\title{
Tanshinol borneol ester on nanostructured lipid carriers has longer brain and systemic effector retention and better antioxidant activity in vivo
}

This article was published in the following Dove Press journal: International Journal of Nanomedicine

\section{Xinyi Yuan \\ Fuhuan Fei \\ Huanmei Sun \\ Chaoni Xiao \\ Xinfeng Zhao \\ Yajun Zhang \\ Xiaohui Zheng}

Key Laboratory of Resource Biology and Biotechnology in Western China, Ministry of Education, College of Life Sciences, Northwest University, Xi'an, People's Republic of China
Correspondence: Yajun Zhang College of Life Sciences, Northwest University, No 229 North Taibai Avenue, Xi'an 710069, People's Republic of China Tel +862988302686 Fax +862988302686 Email zhangyj@nwu.edu.cn
Background: Tanshinol borneol ester (DBZ) is a hybrid of danshensu (DSS) and borneol and has anti-ischemic activity in animals. However, its low water solubility and short half-life limit its clinical application.

Methods: We prepared polyethylene glycol (PEG)-modified and DBZ-loaded nanostructured lipid carriers (DBZ-PEG-NLC) and DBZ-NLC, and examined their physical characteristics, such as particle size, zeta potential, entrapment efficiency and drug loading. The in vitro stability and pharmacokinetics in rats as well as antioxidant activity of DBZ-PEG-NLC and DBZ-NLC in a C57BL/6 mouse model of ischemia/reperfusion-related brain injury were investigated. The levels of DBZ and its hydrolyzed DSS in rat plasma and brain microdialysates were determined by liquid chromatography-mass spectroscopy/mass spectroscopy analysis.

Results: We found that the mean particle size and entrapment efficacy of DBZ-PEG-NLC were similar to that of DBZ-NLC. The DBZ-PEG-NLC, like DBZ-NLC, released DBZ in a biphasic manner with initially burst release and then prolonged slow release in vitro. Intravenous injection of DBZ-PEG-NLC resulted in significantly higher levels and longer retention periods of DBZ and DSS in plasma and the brains than DBZ-NLC and DBZ in rats. Finally, treatment with DBZ-PEG-NLC achieved a better antioxidant activity than DBZ or DBZ-NLC in mouse model of ischemia/reperfusion by reducing the levels of brain malondialdehyde, but increasing the levels of brain superoxide dismutase and glutathione.

Conclusion: DBZ-PEG-NLC is a preferable option to deliver DBZ for sustainable release of DSS and borneol in vivo, and may serve as a promising drug for effective therapy of ischemic cardiovascular and cerebrovascular diseases.

Keywords: nanovesicles, pharmacokinetics, brain microdialysis, ischemic cerebrovascular diseases, polyethylene glycol, danshensu

\section{Introduction}

Tanshinol borneol ester (1,7,7-trimethylbicyclo[2.2.1]heptan-2-yl 3-[3,4dihydroxyphenyl]-2-hydroxy-propanoate [DBZ]) is a hybrid of danshensu (3,4-dihydroxyphenyllactic acid, DSS) and borneol (endo-1,7,7-trimethyl-bicyclo[2.2.1] heptan-2-ol) (Figure 1A). The combination of these two compounds is based on the theory of traditional Chinese medicine and is the formula of Radix Salvia miltiorrhiza and borneol in danshen dropping pills, which have been demonstrated to be effective in the treatment of ischemic cardiovascular and cerebrovascular diseases for years in People's Republic of China. ${ }^{1}$ Previous studies have shown that DBZ is beneficial in patients with cardiovascular and cerebrovascular disease and that its therapeutic effects are better than either DSS or borneol alone. ${ }^{2-5}$ Additionally, DBZ can be conveniently 
A
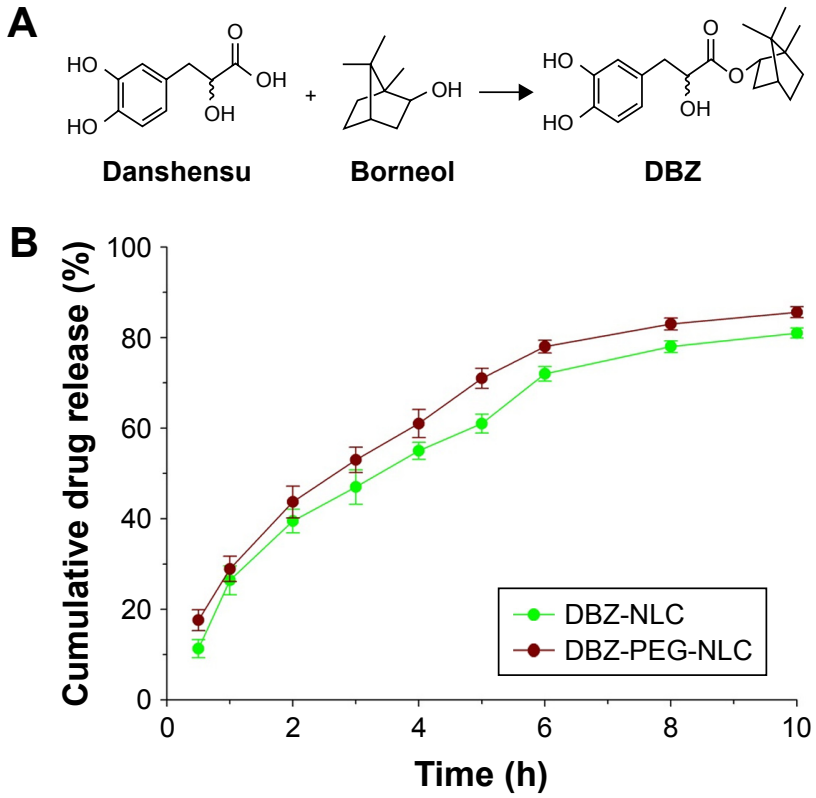

Figure I Chemical structures of DBZ and in vitro release of DBZ from DBZ-PEGNLC and DBZ-NLC.

Notes: (A) Chemical structures of DBZ; (B) in vitro release of DBZ from DBZPEG-NLC and DBZ-NLC. Data are expressed as the mean \pm SD from three separate experiments.

Abbreviations: DBZ, tanshinol borneol ester; DBZ-NLC, DBZ nanostructured lipid carriers; DBZ-PEG-NLC, polyethylene glycol (PEG)-modified and DBZ-loaded nanostructured lipid carriers.

prepared for various dosage forms compared with a mixture of water-soluble DSS and water-insoluble borneol.

However, the therapeutic application of DBZ is limited by its water insolubility and a short half-life. It is notable that the ester bond in DBZ can be readily hydrolyzed to free DSS and borneol by esterases in blood, the liver, and other organs. ${ }^{6}$ Previous studies of metabolism in rat and human liver microsomes indicated that DBZ is metabolized quickly by the liver microsomal enzymes, such as CYP28. ${ }^{7,8}$ Our preliminary pharmacokinetic studies in rats have exhibited that DBZ is eliminated quickly from the blood circulation after injection. Hence, DBZ may require frequent administrations to achieve its therapeutic effect, which may increase its potential side effects. Given that the effective concentrations of a drug in the brain is closely proportional to its plasma concentrations if the drug can effectively pass through the blood-brain barrier, ${ }^{9}$ the rapid elimination of DBZ in plasma should affect its brain delivery. Thus, the development of a new drug formulation is of significance in improving the solubility of DBZ and extending its blood-circulating period.

Nanostructured lipid carriers (NLC) have been demonstrated to be a potentially alternative colloidal drug delivery system due to their fine biocompatibility, biodegradation, high bioavailability, improved solubility, and large-scale production ability. ${ }^{10-13}$ Because polyethylene glycol (PEG) can improve the surface hydrophilicity and prevent the adsorption of lipoproteins and opsonins, modification of NLC with PEG has been used to increase the half-life of the carried drug. . $^{14,15}$ Actually, the PEGylated nanoparticles are able to protect the carried drug from biological and chemical degradation, and have favorable biological properties, including biocompatibility, biodegradability, and long-circulating behavior. ${ }^{16,17}$

In this study, NLC and PEGylated NLC were loaded with DBZ to generate DBZ-NLC and DBZ-PEG-NLC, respectively, and their characteristics were investigated. Considering DSS is also an effector, both DBZ and DSS levels in plasma and the brain were determined and compared following intravenous administration of DBZ, DBZ-NLC, and DBZ-PEG-NLC in rats. Finally, the effects of DBZPEG-NLC on oxidative stress in a mouse model of transient ischemia/reperfusion were evaluated. The results indicated that although both DBZ-NLC and DBZ-PEG-NLC had similar in vitro stability, DBZ-PEG-NLC displayed longer retention periods of DBZ and DSS in rat plasma and brain tissues, associated with better antioxidative activity. Our findings may aid in the design of new types of DBZ drugs for intervention in ischemic cardiovascular diseases.

\section{Materials and methods}

\section{Chemicals and reagents}

DBZ ( $99 \%$ purity) was synthesized in our laboratory, and the chemical structure of DBZ was confirmed by liquid chromatography-mass spectroscopy (LC-MS) and nuclear magnetic resonance spectroscopy. Edaravone (batch number: H20031342) was purchased from Nanjing Simcere Pharma (Jiangsu, People's Republic of China). Glycerol monostearate (GMS) and triglycerides of caprylic/capric acid (MCT) were kindly provided by Gattefosse (Lyon, France). Soybean lecithin (injection grade) was from Lipoid $\mathrm{GmbH}$ (Ludwigshafen, Germany). Pluronic $\mathrm{F}_{68}\left(\mathrm{~F}_{68}\right)$ was from BASF AG (Ludwigshafen, Germany). Tween 80 and PEG-SA (the polymerization degree of ethylene glycol is 40) were obtained from Sigma-Aldrich (St Louis, MO, USA). 4-Hydroxybenzoic acid methyl ester was used as the internal standard in the bioanalysis and obtained from the National Institute for Food and Drug Control (Beijing, People's Republic of China). Sodium pentobarbital was purchased from Beijing Chemical Reagents (Beijing, People's Republic of China). Highperformance liquid chromatography (HPLC)-grade methanol was obtained from Fisher Scientific (Loughborough, UK). Ultrapure water (18.2 M $\Omega$ ) was prepared using a Milli-Q water purification system (Millipore Corporation, Bedford, MA, USA). All other reagents (analytical grade) were purchased from commercial sources. 


\section{Preparation of DBZ-PEG-NLC and DBZ-NLC}

DBZ-PEG-NLC and DBZ-NLC were prepared by emulsion evaporation solidification at a low temperature, and the formulation components including lipids and surfactants were chosen according to the previous reports. ${ }^{11,18}$ Based on the preliminary tests, the orthogonal design method was used to optimize the formulation. The encapsulation efficiency (EE\%), loading capacity (LC\%), and particle size were taken as evaluation indexes. With the amount of DBZ (mg), the ratio of lipoids to lecithin (w/w), and the content of surfactants (\%) as research objects, the optimized formulation of DBZ-PEG-NLC was obtained by $\mathrm{L}_{9}\left(3^{4}\right)$ orthogonal experiment. Briefly, 30 mg DBZ, 375 mg lipids (GMS:PEG-SA: $\mathrm{MCT}=2: 2: 1, \mathrm{w} / \mathrm{w} / \mathrm{w}$ ), and $400 \mathrm{mg}$ lecithin were dissolved in $10 \mathrm{~mL}$ dichloromethane-ethanol $(3: 2, \mathrm{v} / \mathrm{v})$ at $75^{\circ} \mathrm{C}$ in a water bath. Simultaneously, $30 \mathrm{~mL}$ of aqueous solution containing $1.5 \% \mathrm{~F}_{68}$ and tween $80(4: 3, \mathrm{w} / \mathrm{w})$ was heated at the same temperature. The obtained organic phase of DBZ was added dropwise into the aqueous solution and mixed at 13,000 rpm for $30 \mathrm{~min}$ in a high-performance homogenizer (FA25 Model, Fluko Equipment, Shanghai, People's Republic of China), followed by stirring at $2,000 \mathrm{rpm}$ at $75^{\circ} \mathrm{C}$ for $2 \mathrm{~h}$. The generated warm nanoemulsion was quickly solidified in an ice bath $\left(0-2^{\circ} \mathrm{C}\right)$ under stirring at $1,000 \mathrm{rpm}$ for $1 \mathrm{~h}$ to acquire the DBZ-PEG-NLC dispersions. Similarly, the DBZ-NLC dispersions were prepared by replacing PEG with an equivalent amount of GMS. In addition, $1.5 \mathrm{mg} / \mathrm{mL} \mathrm{DBZ}$ was prepared by dissolving DBZ in saline containing 3.0\% $\mathrm{F}_{68}$ for intravenous injection.

\section{Characterization of DBZ-PEG-NLC and DBZ-NLC}

The particle size, polydispersity index (PI), and zeta potential of DBZ-PEG-NLC and DBZ-NLC were measured by photon correlation spectroscopy using a Zetasizer Nano ZS90 (Malvern Instruments, Malvern, UK) at a measurement angle of $90^{\circ}$ and a temperature of $25^{\circ} \mathrm{C}$. All samples were diluted with distilled water and adjusted to a suitable intensity.

The EE and LC of DBZ-PEG-NLC and DBZ-NLC were determined by centrifugal ultrafiltration. ${ }^{19,20}$ Briefly, $0.5 \mathrm{~mL}$ of DBZ-PEG-NLC or DBZ-NLC dispersions were added into one Ultra-4 ultrafiltration tube (molecular weight cut-off was $10 \mathrm{~K}$, Millipore) and centrifuged at $814 \times \mathrm{g}$ for $15 \mathrm{~min}$. The filtrates were collected and analyzed with HPLC as $W_{\text {free }}$. Meanwhile, the DBZ-PEG-NLC or DBZ-NLC dispersions $(0.5 \mathrm{~mL} /$ tube $)$ were treated with $3 \mathrm{~mL}$ of methanol, sonicated for $10 \mathrm{~min}$ to release drug, and centrifuged at $13,034 \times \mathrm{g}$ for $5 \mathrm{~min}$. The liquid phase was collected and analyzed by
HPLC as $W_{\text {total }}$. The DBZ samples were analyzed by HPLC in a liquid chromatographic system with a diode array detector (Agilent Technologies, Santa Clara, CA, USA) at $30^{\circ} \mathrm{C}$ in a $_{18}$ column $(250 \times 4.6 \mathrm{~mm}, 5 \mu \mathrm{m}$; Agilent $)$ using a mobile phase of methanol and $0.2 \%$ formic acid solution $(75: 25, \mathrm{v} / \mathrm{v})$ at a flow rate of $0.8 \mathrm{~mL} / \mathrm{min}$ and ultraviolet detection at $280 \mathrm{~nm}$. The EE values were calculated using the formula $\left(W_{\text {total }}-W_{\text {free }}\right) / W_{\text {total }}$, and LC values were calculated with the formula $\left(W_{\text {total }}-W_{\text {free }}\right) / W_{\text {lipid }} W_{\text {lipid }}$ denotes the weight of lipid added in the system.

\section{In vitro release assay}

The in vitro release of DBZ from the DBZ-PEG-NLC and DBZ-NLC was investigated by dialysis. ${ }^{18,21}$ In brief, the DBZ-PEG-NLC and DBZ-NLC dispersions were dialyzed against $15 \%$ ethanol in PBS $(200 \mathrm{~mL}, \mathrm{pH} 7.4)$ in a dialysis bag (molecular weight cut-off of 10K, Xitang, Shanghai, People's Republic of China) under continual stirring at $100 \mathrm{rpm}$ at $37^{\circ} \mathrm{C}$. The PBS samples $(1 \mathrm{~mL})$ were collected in triplicate longitudinally at $0.5,1,2,3,4,5,6,8$, and $10 \mathrm{~h}$ and determined by HPLC.

\section{Pharmacokinetics analysis in rats}

Adult male Sprague Dawley (SD) rats (220-250 g) and male C57BL/6 mice (18-22 g) were obtained from the Experimental Animal Center of Xi'an Jiaotong University. The animals were housed in a specific pathogen-free facility with free access to food and water. The animal studies were carried out in accordance with the guidelines regarding the principles of animal care of the National Institute of Health (People's Republic of China, 2004) and approved by the Animal Care and Use Committee of Northwest University in People's Republic of China.

SD rats were fasted overnight, randomized, and injected intravenously with $6 \mathrm{mg} / \mathrm{kg}$ of DBZ-PEG-NLC, DBZ-NLC, or DBZ ( $\mathrm{n}=6$ per group). ${ }^{2}$ Their blood samples were collected using ABS2 automated blood collector (Instech Laboratories, Plymouth Meeting, PA, USA) at 2, 5, 10, 20, 30, 45, $60,90,180$, or 300 min postinjection and injected with the same volume of saline to maintain total blood volume after collection of each sample. The blood samples were centrifuged to prepare plasma under a low temperature ${ }^{22}$ and stored at $-80^{\circ} \mathrm{C}$.

\section{Evaluation of brain delivery by the microdialysis method}

\section{Probe implantation}

The rats were shaved to expose their skulls, anesthetized, and placed in a stereotaxic apparatus. A midline incision 
of $\sim 2 \mathrm{~cm}$ along the sagittal suture and the bregma point was made in individual rat skulls and used as the reference point for positioning the microdialysis probe. Individual rats were inserted stereotaxically with a microdialysis probe (DM-22, $200 \mu \mathrm{m}$ inner diameter and $220 \mu \mathrm{m}$ outer diameter; Eicom, Kyoto, Japan) that was filled with artificial cerebrospinal solution (128 mM NaCl, $2.6 \mathrm{mM} \mathrm{KCl,} 1.26 \mathrm{mM} \mathrm{CaCl}_{2}$, and $2 \mathrm{mM} \mathrm{MgCl}_{2}$ ) through a cranial burr hole of $3.1 \mathrm{~mm}$ depth, $1.5 \mathrm{~mm}$ lateral, and $0.9 \mathrm{~mm}$ posterior of the bregma point, and the probe was attached to the skull with dental cement.

\section{Microdialysis procedure}

The inlet tubing of the microdialysis probe was connected with a microinjection pump (EFC-82, Eicom) and perfused with the artificial cerebrospinal fluid beginning at a rate of $5 \mu \mathrm{L} / \mathrm{min}$ for $5 \mathrm{~min}$ and changed to $1.5 \mu \mathrm{L} / \mathrm{min}$ throughout the experiment. After recovery for $1 \mathrm{~h}$, individual rats were injected intravenously with $6 \mathrm{mg} / \mathrm{kg}$ of DBZ-PEGNLC, DBZ-NLC, and DBZ. All perfusates were collected continually and sampled in fractions every 20 min up to $140 \mathrm{~min}$. The in vivo recovery was measured by the retrodialysis. The recovery rates were $40.62 \%, 41.79 \%$, and $41.38 \%$ for DBZ and $39.21 \%, 38.15 \%$, and $37.62 \%$ for DSS, respectively.

\section{Determination of DBZ and DSS concentrations by LC-MS/MS}

The DBZ can be transformed to DSS by esterase decomposition in plasma and the brain following intravenous administration. Both DBZ and hydrolyzed DSS in plasma and the microdialysates were determined by LC-MS. Individual plasma samples $(100 \mu \mathrm{L})$ were mixed with $500 \mu \mathrm{L}$ of ice-cold acetonitrile and $10 \mu \mathrm{L}$ internal standard (4-hydroxybenzoic acid methyl ester solution in methanol, $10 \mu \mathrm{g} / \mathrm{mL}$ ). The mixtures were vortexed for $5 \mathrm{~min}$, and centrifuged at $4^{\circ} \mathrm{C}, 2,000 \times g$ for $5 \mathrm{~min}$. The supernatant was collected and dried under a stream of nitrogen. The residues were reconstituted in $100 \mu \mathrm{L}$ of methanol and $20 \mu \mathrm{L}$ of each was injected for LC-MS analysis. The perfusate samples (20 $\mu \mathrm{L}$ each) were directly analyzed by LC-MS.

The LC-MS was performed in the Waters Quattro Micro API mass spectrometer coupled to an Alliance HT HPLC system (Waters, Milford, MA, USA) in an Atlantis $\mathrm{C}_{18}$ column $(150 \times 4.6 \mathrm{~mm}, 5 \mu \mathrm{m})$ with a mobile phase of methanol and $0.5 \%$ formic acid solution $(60: 40, \mathrm{v} / \mathrm{v})$ at a flow rate of $0.6 \mathrm{~mL} / \mathrm{min}$. To optimize MS-MS conditions, the daughter ion spectrum of the $[\mathrm{M}-\mathrm{H}]^{-}$ion was recorded by ramping up the capillary voltage and the collision energy. The most abundant fragments were detected at $\mathrm{m} / z 197.2$ for DBZ, $m / z 179.1$ for DSS, and $m / z 136.3$ for internal standard with a capillary voltage of $2,500 \mathrm{~V}$ and a collision energy of $18 \mathrm{eV}$, respectively. The amounts of each compound were quantitatively analyzed using the multiple reaction monitoring mode by calculating the ratios of $\mathrm{m} / \mathrm{z} 333.2$ (parent ion) to $m / z 197.2$ (product ion) for DBZ, $m / z 197.2$ (parent ion) to 179.1 (product ion) for DSS, and $m / z 151.2$ (parent ion) to $m / z 136.3$ (production) for the IS. The main partial validation parameters of the analytical method employed are summarized in Table 1.

\section{Evaluation of DBZ-PEG-NLC bioactivity in a mouse model of transient cerebral ischemic/reperfusion injury}

Transient cerebral ischemia/reperfusion model and treatment

C57BL/6 mice were used for establishment of transient cerebral ischemic/reperfusion injury. ${ }^{23,24}$ Briefly, individual mice were injected intraperitoneally with sodium pentobarbital $(60 \mathrm{mg} / \mathrm{kg})$. Their right and left carotid arteries were exposed and clamped for 20 min using vascular clamps (time of ischemia). The vascular clamps were removed to allow reperfusion of blood flow. Immediately after reperfusion, the operated mice were randomized and injected intravenously with vehicle saline $(100 \mu \mathrm{L})$ as the control, 6 and $12 \mathrm{mg} / \mathrm{kg}$ of DBZ, DBZ-NLC, DBZ-PEG-NLC or $4 \mathrm{mg} / \mathrm{kg}$ of Edaravone (the positive control). A sham group of mice received the same procedure without carotid artery clamping $(n=10$ per group). Animals were kept in an air-ventilated incubator at

Table I HPLC-MS quantification of DBZ and DSS in plasma and microdialysate $(n=5)$

\begin{tabular}{llll}
\hline Drug & Validation parameters & Plasma & Microdialysate \\
\hline DBZ & Calibration range $(\mu \mathrm{g} / \mathrm{mL})$ & $0.045-4.5$ & $0.02-1.0$ \\
& Coefficient of determination $\left(r^{2}\right)$ & 0.996 & 0.999 \\
& LOQ $(\mu \mathrm{g} / \mathrm{mL})$ & 0.01 & 0.015 \\
& Precision (interday, RSD\%) & $\leq 7.85$ & $\leq 2.27$ \\
& Accuracy $(\mathrm{RE} \%)$ & $-1.41-1.53$ & $0.28-1.55$ \\
DSS & Calibration range $(\mu \mathrm{g} / \mathrm{mL})$ & $0.045-4.5$ & $0.02-1.0$ \\
& Coefficient of determination $\left(r^{2}\right)$ & 0.993 & 0.999 \\
& LOQ $(\mu g / \mathrm{mL})$ & 0.01 & 0.015 \\
& Precision (interday, RSD\%) & $\leq 9.22$ & $\leq 2.64$ \\
& Accuracy (RE\%) & $-3.26-1.77$ & $0.29-1.47$ \\
\hline
\end{tabular}

Abbreviations: DBZ, tanshinol borneol ester; DSS, danshensu; HPLC-MS, highperformance liquid chromatography-mass spectroscopy; LOQ, limit of quantification; RE, relative error; RSD, relative SD. 
$24.0^{\circ} \mathrm{C} \pm 0.5^{\circ} \mathrm{C}$ and sacrificed at $24 \mathrm{~h}$ after cerebral ischemia. Their brain tissues were dissected out and kept at $-60^{\circ} \mathrm{C}$ until biochemical assessment.

\section{Biochemical analyses}

Individual brain tissue samples, except for the cerebellum and brainstem, were homogenized in 10 volume $(\% \mathrm{w} / \mathrm{v})$ of PBS (100 mM, pH 7.4) and centrifuged (12,000×g, $15 \mathrm{~min}$, $4^{\circ} \mathrm{C}$ ). The supernatants were collected for determination of the levels of malondialdehyde (MDA), superoxide dismutase (SOD), and glutathione (GSH) by enzyme-linked immunosorbent assay using specific kits (Elabscience, Wuhan, People's Republic of China), according to the manufacturer's instructions.

\section{Data analysis}

The pharmacokinetic parameters in plasma were estimated from the mean concentration values using noncompartmental pharmacokinetic analysis with the Das 2.0 program (Mathematical Pharmacology Professional Committee of China, Shanghai, People's Republic of China). All data are expressed as mean $\pm \mathrm{SD}$. The difference among the groups was analyzed by one-way analysis of variance or Student's $t$-test. All statistical analyses were performed using the SPSS 18.0 program (SPSS Inc., Chicago, IL, USA). A $p$-value of $<0.05$ was considered statistically significant.

\section{Results}

\section{Characterizations of DBZ-PEG-NLC and DBZ-NLC}

To generate more stable and efficient DBZ drugs, DBZ-PEGNLC and DBZ-NLC were prepared by emulsion evaporation solidification at a low temperature (Figure 1A). Characterization indicated that the mean particle sizes of DBZ-PEG-NLC and DBZ-NLC were 181.3 \pm 5.6 and $194.1 \pm 6.3 \mathrm{~nm}$, while their PIs were $0.20 \pm 0.028$ and $0.22 \pm 0.035$, respectively (Table 2). Such data indicated that both DBZ-PEG-NLC and DBZ-NLC had similar PIs (PI $<0.25)$. Further analysis revealed that the zeta potentials of DBZ-PEG-NLC and DBZNLC were $-10.4 \pm 0.49$ and $-17.6 \pm 0.65 \mathrm{mV}$, respectively. In general, the zeta potential of colloidal carriers is above the critical value of $-30 \mathrm{mV}$, implying the good stability. ${ }^{25} \mathrm{In}$ this study, the zeta potentials of both DBZ-NLC and DBZPEG-NLC were below the critical value. Because the steric stability layer was composed of $\mathrm{F}_{68}$ in DBZ-NLC, and $\mathrm{F}_{68}$ and PEG chains in DBZ-PEG-NLC, nanoparticles could still have long-term stability. ${ }^{14}$ HPLC assay demonstrated
Table 2 The physical characteristics of DBZ-PEG-NLC and DBZ-NLC

\begin{tabular}{llllll}
\hline Sample & $\begin{array}{l}\text { Particle } \\
\text { size } \\
(\mathbf{n m})\end{array}$ & $\begin{array}{l}\text { PI } \\
\text { potential } \\
(\mathbf{m V})\end{array}$ & EE (\%) & LC (\%) \\
\hline DBZ-PEG- & $181.3 \pm 5.6$ & $0.20 \pm 0.028$ & $-10.42 \pm 0.49$ & $78.72 \pm 3.61$ & $6.15 \pm 0.28$ \\
NLC & & & & & \\
DBZ-NLC & $194.1 \pm 6.3$ & $0.22 \pm 0.035$ & $-17.60 \pm 0.65$ & $81.35 \pm 4.27$ & $6.32 \pm 0.33$ \\
\hline
\end{tabular}

Note: Data are expressed as the mean \pm SD from three separate experiments. Abbreviations: DBZ, tanshinol borneol ester; DBZ-NLC, DBZ nanostructured lipid carriers; DBZ-PEG-NLC, polyethylene glycol (PEG)-modified and DBZ-loaded nanostructured lipid carriers; EE, encapsulation efficiency; LC, loading capacity; $\mathrm{PI}$, polydisperity index.

that the EE values of DBZ-PEG-NLC and DBZ-NLC were $78.72 \% \pm 3.61 \%$ and $81.35 \% \pm 4.27 \%$, while the $\mathrm{LC}$ values of DBZ-PEG-NLC and DBZ-NLC were $6.15 \% \pm 0.28 \%$ and $6.32 \% \pm 0.33 \%$, respectively. Hence, both DBZ-PEGNLC and DBZ-NLC had good drug entrapment efficiency and LC.

To test the in vitro stability, DBZ-PEG-NLC and DBZNLC were dialyzed against $15 \%$ ethanol PBS and the released DBZ was measured longitudinally by HPLC. As shown in Figure 1B, both DBZ-PEG-NLC and DBZ-NLC displayed a biphasic pattern of DBZ release in vitro. Approximately $45 \%$ of total DBZ was rapidly released from both DBZ-PEG-NLC and DBZ-NLC during the first 2-h period, while the DBZ release was slowed and almost $86 \%$ of the DBZ was released at $10 \mathrm{~h}$. Although the DBZ-PEG-NLC had slightly higher levels of DBZ release, there was no significant difference in the in vitro stability between DBZ-PEG-NLC and DBZ-NLC in our experimental conditions. Taken together, addition of PEG does not affect the physical characteristics and in vitro stability of DBZ-NLC.

\section{Pharmacokinetics of DBZ-PEG-NLC and DBZ-NLC in rats following intravenous administration}

Next, we tested the pharmacokinetics of DBZ-PEG-NLC and DBZ-NLC in rats. SD rats were injected intravenously with $6.0 \mathrm{mg} / \mathrm{kg}$ DBZ, DBZ-PEG-NLC, or DBZ-NLC and plasma samples collected. The levels of plasma DBZ in individual rats were measured longitudinally by LC-MS (Figure 2A). The concentrations of plasma DBZ displayed as a two-compartment model, an initial rapid decrease in the concentration of DBZ and a slower terminal elimination of DBZ. The peak concentrations of $\mathrm{DBZ}$ in the rats receiving DBZ-PEG-NLC or DBZ-NLC were higher than that in the rats with DBZ. Although the concentrations of DBZ in all rats 

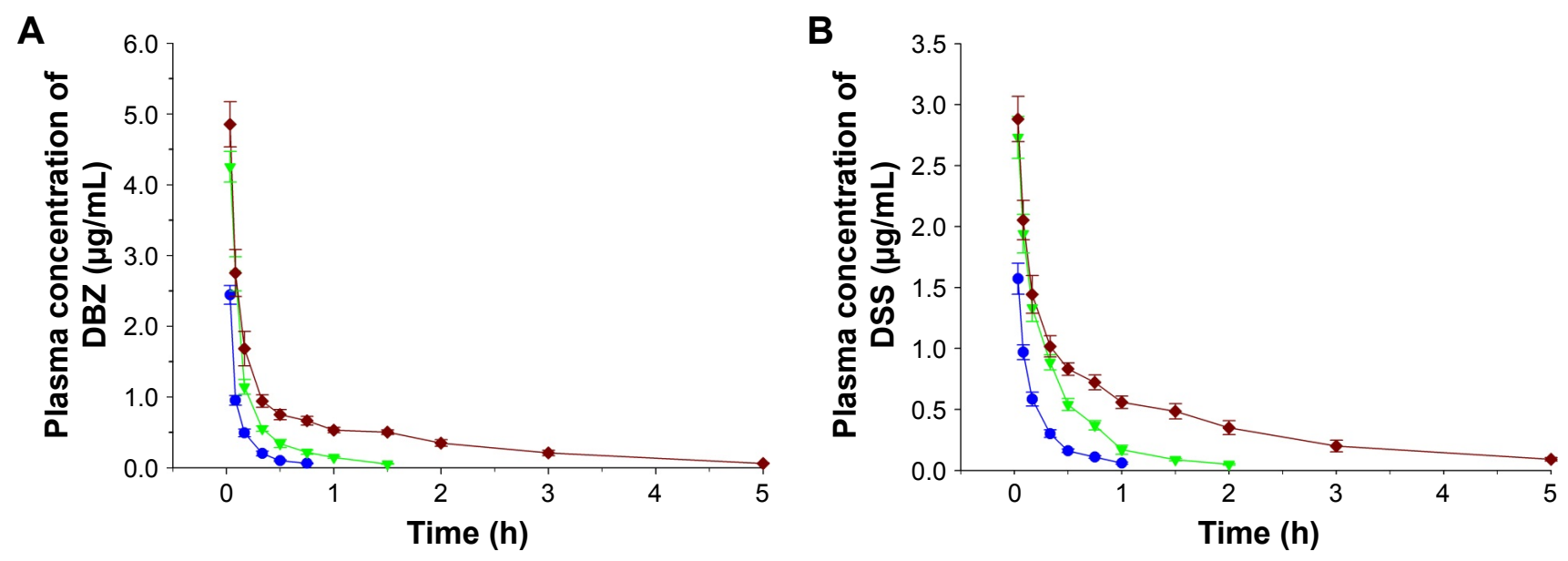

$\bullet$ DBZ $\rightarrow$ DBZ-NLC $\rightarrow$ DBZ-PEG-NLC

Figure 2 The levels of plasma DBZ and DSS.

Notes: (A) The levels of plasma DBZ; (B) the levels of plasma DSS. Data are expressed as the mean \pm SD from each group ( $\mathrm{n}=6$ per group) for three separate experiments.

Abbreviations: DBZ, tanshinol borneol ester; DBZ-NLC, DBZ nanostructured lipid carriers; DBZ-PEG-NLC, polyethylene glycol (PEG)-modified and DBZ-loaded nanostructured lipid carriers; DSS, danshensu.

rapidly decreased within the first $15 \mathrm{~min}$, the levels of DBZ in the rats that had been injected with DBZ-PEG-NLC were higher than that of the other two groups. Quantitative analysis indicated that the area under the plasma concentration-time curve and mean residency time values of DBZ in the DBZPEG-NLC group were significantly higher than that of the DBZ-NLC group, which were higher than that of the DBZ group (Table 3). In contrast, the clearance values of DBZ in the DBZ-PEG-NLC group were less than that of the DBZNLC group, which were also less than that of the DBZ group. Similar patterns of plasma DSS were detected among these groups of rats (Figure 2B; Table 3). Taken together, the DBZPEG-NLC had longer plasma retention periods of DBZ and DSS in rats following intravenous injection.

\section{The concentrations of DBZ and DSS in the brains of rats following intravenous administration of DBZ-PEG-NLC and DBZ-NLC}

Given that the concentrations of DBZ and DSS in the central nervous system are crucial for their therapeutic effects, we determined the concentrations of DBZ and DSS in the brain following delivery of DBZ, DBZ-NLC, and DBZ-PEG-NLC in rats by microdialysis system for sampling. Although the concentrations of DBZ in the brain from the three groups of rats were similar during the first 20 min post drug delivery, the concentrations of DBZ in the brain from the DBZ-PEGNLC group of rats were significantly higher than that of

Table 3 The pharmacokinetic parameters of plasma DBZ and DSS

\begin{tabular}{|c|c|c|c|c|c|}
\hline Drug & Parameter & Unit & DBZ & DBZ-NLC & DBZ-PEG-NLC \\
\hline \multirow[t]{4}{*}{ DBZ } & $\mathrm{AUC}_{0-\infty}$ & $\mathrm{mg} / \mathrm{L} \mathrm{h}$ & $0.392 \pm 0.043$ & $0.869 \pm 0.107^{a}$ & $2.29 I \pm 0.334^{\mathrm{a}, \mathrm{b}}$ \\
\hline & $V_{d}$ & $\mathrm{~L} / \mathrm{kg}$ & $3.387 \pm 0.463$ & $3.557 \pm 0.415$ & $4.209 \pm 0.486$ \\
\hline & $\mathrm{CL}$ & L/h kg & $16.14 \mid \pm 2.135$ & $6.903 \pm 0.882^{\mathrm{a}}$ & $2.5 \mid 2 \pm 0.20 \mathrm{I}^{\mathrm{a}, \mathrm{b}}$ \\
\hline & $\mathrm{MRT}_{0-\infty}$ & $\mathrm{H}$ & $0.149 \pm 0.015$ & $0.316 \pm 0.043^{a}$ & $1.301 \pm 0.152^{\mathrm{a}, \mathrm{b}}$ \\
\hline \multirow[t]{4}{*}{ DSS } & $\mathrm{AUC}_{0-\infty}$ & $\mathrm{mg} / \mathrm{L} \mathrm{h}$ & $0.403 \pm 0.04 I$ & $0.938 \pm 0.13 \mathrm{I}^{\mathrm{a}}$ & $2.266 \pm 0.218^{\mathrm{a}, \mathrm{b}}$ \\
\hline & $V_{d}$ & $\mathrm{~L} / \mathrm{kg}$ & $6.498 \pm 0.918$ & $5.395 \pm 0.735$ & $5.389 \pm 0.885$ \\
\hline & $\mathrm{CL}$ & L/h kg & $|4.658 \pm 2.5| 4$ & $6.405 \pm 0.798^{\mathrm{a}}$ & $2.606 \pm 0.276^{\mathrm{a}, \mathrm{b}}$ \\
\hline & $M R T_{0-\infty}$ & $\mathrm{H}$ & $0.305 \pm 0.045$ & $0.516 \pm 0.067^{\mathrm{a}}$ & $1.749 \pm 0.239^{a, b}$ \\
\hline
\end{tabular}

Notes: Data are expressed as the mean \pm SD of each group $(n=6)$ from three separate experiments following intravenous administration of DBZ, DBZ-NLC, or DBZ-PEGNLC at a dose of $6 \mathrm{mg} / \mathrm{kg}$. ${ }^{a} p<0.05$ vs the DBZ; ${ }^{b} p<0.05$ vs the DBZ-NLC.

Abbreviations: AUC, area under the plasma concentration-time curve; CL, clearance; DBZ, tanshinol borneol ester; DBZ-NLC, DBZ nanostructured lipid carriers; DBZ-PEG-NLC, polyethylene glycol (PEG)-modified and DBZ-loaded nanostructured lipid carriers; DSS, danshensu; MRT $_{0-\infty}$, mean residency time; $V_{\mathrm{d}}$, volume of body distribution. 


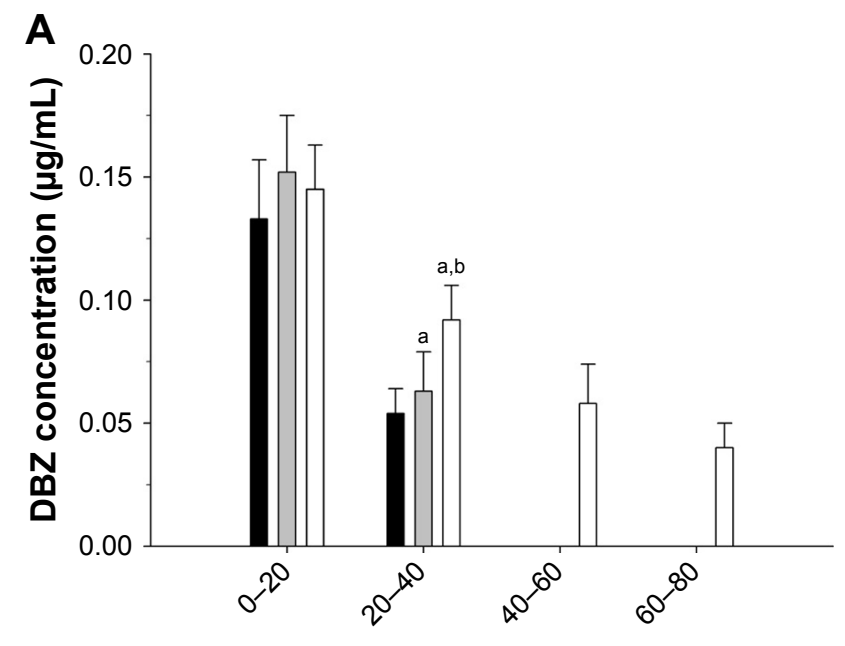

Time (min)

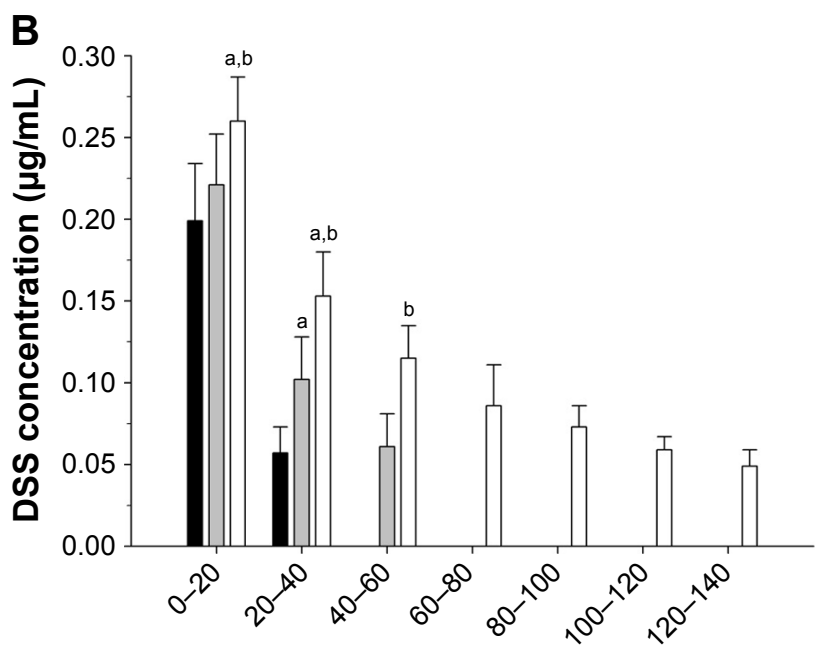

Time (min)

DBZ $\square$ DBZ-NLC $\square$ DBZ-PEG-NLC

Figure 3 The levels of DBZ and DSS in the brains of rats.

Notes: (A) The levels of DBZ in the brains; (B) the levels of DSS in the brains. Data are expressed as the mean \pm SD from each group ( $n=6$ per group) for three separate experiments. ${ }^{a} p<0.01$ vs the DBZ group. ${ }^{b} p<0.01$ vs the DBZ-NLC group.

Abbreviations: DBZ, tanshinol borneol ester; DBZ-NLC, DBZ nanostructured lipid carriers; DBZ-PEG-NLC, polyethylene glycol (PEG)-modified and DBZ-loaded nanostructured lipid carriers; DSS, danshensu.

other groups of rats between 20 and 40 min postinjection (Figure 3A). Furthermore, there were detectable levels of DBZ in the brains from the DBZ-PEG-NLC group of rats, but not from other groups of rats at 60 and 80 min postinjection. Further analysis revealed that the levels of DSS in the brains were significantly higher than that of DBZ in all groups of rats (Figure 3B). The concentrations of DSS in the brains from the DBZ-PEG-NLC group of rats were significantly higher than that in the other groups of rats during the first and second 20 min postinjection. While brain DSS was detected during 40-60 min in the DBZ group of rats and 40-60 min in the DBZ-NLC group of rats, it was detected at later time periods in the DBZ-PEG-NLC group of rats. Such data demonstrated that DBZ-PEG-NLC treatment maintained higher concentrations and longer retention periods of DBZ and DSS in the brain of rats.

\section{DBZ-PEG-NLC has a better antioxidant activity in mouse model of cerebral ischemic/reperfusion}

Finally, we tested the effect of treatment with DBZ, DBZNLC, or DBZ-PEG-NLC on oxidative stress in a mouse model of cerebral ischemia/reperfusion. C57BL/6 mice were induced for cerebral ischemia reperfusion. The mice were randomized and intravenously injected with vehicle (the control), 6 or $12 \mathrm{mg} / \mathrm{kg}$ DBZ, or equal molecular weight DBZ-NLC or DBZ-PEG-NLC, or positive control $4 \mathrm{mg} / \mathrm{kg}$ Edaravone immediately after reperfusion. A group of mice received the sham surgery. The levels of brain MDA, SOD, and GSH in individual mice were determined by enzyme-linked immunosorbent assay. In comparison with the sham group of mice, significantly higher levels of MDA and lower levels of SOD and GSH were detected in the brains of the control mice $(P<0.01$ for all, Figure $4 \mathrm{~A}-\mathrm{C})$. Treatment with DBZ or DBZ-NLC at $12 \mathrm{mg} / \mathrm{kg}$ significantly decreased the levels of MDA, but increased the levels of SOD and GSH in the brains of mice $(P<0.05$ for all $)$, relative to that in the control mice. Furthermore, the therapeutic effects of DBZ-PEGNLC were better than that of DBZ and DBZ-NLC, and were slightly less than that of Edaravone in this model. Therefore, DBZ-PEG-NLC has a better antioxidant activity than DBZ and DBZ-NLC in this model.

\section{Discussion}

DSS and borneol are bioactive components and have potent antioxidant ${ }^{26}$ and antiplatelet aggregation effects, ${ }^{27}$ improving ischemia and reperfusion injury. ${ }^{28-31}$ Furthermore, borneol can improve the blood-brain barrier permeability and enhance the distribution and therapeutic effect of brain-protecting drugs. ${ }^{32,33}$ DBZ, a hybrid of DSS and borneol, has shown better therapeutic effects. However, due to its poor solubility and a short half-life, its therapeutic 

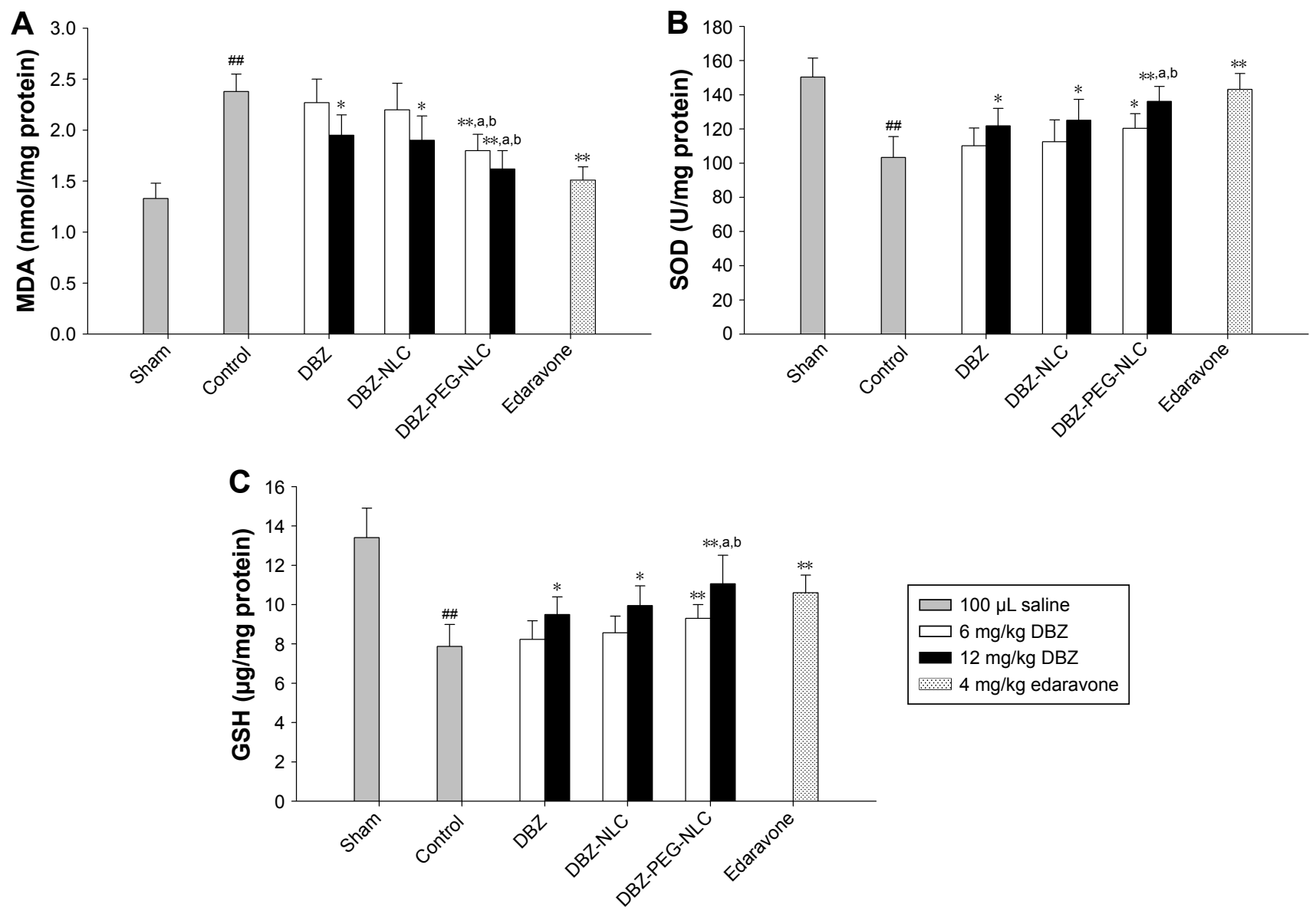

Figure 4 Treatment with DBZ-PEG-NLC improves oxidative stress in the brain of a mouse model of ischemia/reperfusion-related injury.

Notes: (A) The levels of MDA in the brains; (B) the levels of SOD in the brains; (C) the levels of GSH in the brain. Data are expressed as the mean \pm SD from each group ( $n=10$ per group) for three separate experiments. ${ }^{\#} p<0.01$ vs the sham-operated group. ${ }^{*} p<0.05,{ }^{* *} p<0.01$ vs the control group. ${ }^{a} p<0.05$ vs the $D B Z$ group and ${ }^{b} p<0.05$ vs the DBZ-NLC group.

Abbreviations: DBZ, tanshinol borneol ester; DBZ-NLC, DBZ nanostructured lipid carriers; DBZ-PEG-NLC, polyethylene glycol (PEG)-modified and DBZ-loaded nanostructured lipid carriers; GSH, glutathione; MDA, malondialdehyde; SOD, superoxide dismutase.

application is limited in the clinic. It is notable that NLC have many advantages to effectively deliver compounds with high EE and LC, and it can be eliminated by endogenous macrophages. ${ }^{34,35}$ The PEGylated nanoparticles are able to protect the carried drug from biological and chemical degradation, increasing the systemic retention periods of the drug in vivo. ${ }^{36}$

In this study, we generated DBZ-NLC and DBZ-PEGNLC and found that both DBZ-NLC and DBZ-PEG-NLC had similar PIs and good drug entrapment efficiency and LC. In addition, both DBZ-PEG-NLC and DBZ-NLC displayed a biphasic pattern of DBZ release in vitro. Such data indicated that addition of PEG did not affect physical characteristics and in vitro stability of DBZ-NLC.

After intravenous administration, the NLCs that were composed of lipids and lecithin would be degraded gradually in blood or organs. It is possible that the both NLC formulations in the presence of plasma proteins would not retain their structures prior to drug release. However, there are other primary concerns for NLCs following their injection. The nanocarriers may interact with plasma proteins to form protein corona, recognized and eliminated by macrophages in the reticuloendothelial system, which are responsible for the biological fate of nanocarriers. ${ }^{35,37}$ The composition of the protein corona and the process of cellular uptake are still unclear and may be complex. ${ }^{17}$

In the present study, the in vivo evaluation of DBZ-PEGNLC was carried out by pharmacokinetic experimentation on DBZ and DSS. The results revealed that while intravenous injection with DBZ resulted in rapid clearance of plasma DBZ and DSS in rats, intravenous administration of DBZPEG-NLC significantly prolonged the retention periods of plasma DBZ and DSS with significantly higher area under the plasma concentration-time curve and mean residency time values of DBZ than that of the DBZ-NLC. Similarly, we employed a microdialysis system to investigate the distribution of drugs in the brain. ${ }^{38} \mathrm{We}$ found that intravenous administration of DBZ-PEG-NLC resulted in significantly 
higher levels and longer retention periods of DSS and DBZ in the brain of rats compared with the rats injected with $\mathrm{DBZ}$ or DBZ-NLC. The high levels of DSS in the brain may be from rapid conversion of DBZ to DSS in the brain because the levels of plasma DSS were lower than that of DBZ in those rats. The longer retention periods of DBZ and DSS may stem from longer plasma retention of DBZ and DSS. The high levels of bioactive DSS suggest that treatment with DBZ-PEG-NLC may have a better therapeutic effect on cerebral ischemia/reperfusion-related injury. Given that DBZ can be hydrolyzed to free DSS and borneol by esterases in blood or other organs, the longer retention periods of DBZ and DSS suggest a long retention period of plasma borneol in rats, which should enhance their bioactivity against ischemia/ reperfusion-related injury.

Oxidative stress can result in high levels of reactive oxygen species (ROS) production and promote inflammatory cytokine secretion, contributing to the pathogenesis of ischemic stroke. ${ }^{39}$ The high levels of ROS can damage the structure of long-chain polyunsaturated fatty acids and cause their peroxidation in the brain, damaging the neurons in the central nervous system. ${ }^{40,41}$ Actually, treatment with Edaravone, a free radical scavenger, benefits patients with acute stroke. ${ }^{42}$ In this study, we found that treatment with DBZ, DBZ-NLC, or DBZ-PEG-NLC immediately after reperfusion significantly decreased the levels of brain MDA, but increased the levels of SOD and GSH in a mouse model of cerebral ischemia/reperfusion-related injury. Although the therapeutic effect of DBZ-PEG-NLC was slightly less than that of Edaravone, it was significantly higher than that of DBZ or DBZ-NLC in this model. The stronger therapeutic effect of DBZ-PEG-NLC may be associated with its longer retention periods of DBZ and DSS in the brain. Mechanistically, the active compounds of DBZ, DSS, and borneol may inhibit lipid peroxidation and improve the activity of SOD and GSH levels to reduce oxidative stress and MDA levels in the brain. In this acute ischemic model, we did not observe any obvious difference in mouse brain morphology among the groups of mice, although DBZ-PEG-NLC had a better antioxidant activity. We are interested in further investigating potential toxicity of the prolonged and higher levels of DBZ and DSS, therapeutic effect, and the molecular mechanisms underlying the action of DBZ-PEG-NLC in other animal models of ischemia/reperfusion-related brain injury.

\section{Conclusion}

In summary, our data indicated that both DBZ-NLC and DBZPEG-NLC had similar physical characteristics and in vitro stability. Intravenous administration of DBZ-PEG-NLC resulted in prolonger retention periods of DBZ and DSS in plasma and the brain than DBZ or DBZ-NLC in rats. Treatment with DBZ-PEG-NLC had better therapeutic effect in reducing oxidative stress in the brain of a mouse model of ischemia/reperfusion-related injury. Therefore, DBZ-PEGNLC may be a promising drug for intervention in ischemic cardiovascular and cerebrovascular diseases.

\section{Acknowledgments}

This study was supported by grants from the Changjiang Scholars and Innovative Research Team in University (No IRT_15R55), and the Natural Science Basic Research Plan in Shaanxi Province of People's Republic of China (No 2016JM8057).

\section{Disclosure}

The authors report no conflicts of interest in this work.

\section{References}

1. Zhao X, Zheng X, Fan TP, Li Z, Zhang Y, Zheng J. A novel drug discovery strategy inspired by traditional medicine philosophies. Science. 2015;347:S38-S40.

2. Liu M, Sun JN, Zhang SF, et al. Protective effect of tanshinol borneol ester on rats with middle cerebral artery occlusion. Chin Pharmacol Bull. 2009;25(Suppl):301-303.

3. Liao S, Fan TP, Zhao XF, Choi R, Zheng X. DBZ, a novel synthetic compound inspired by Compound Danshen Dripping Pills, promotes angiogenesis in vitro through activating Akt and MAPK signalling pathways. Eur J Integr Med. 2014;6(6):688.

4. Jia P, Wang SX, Xiao CN, et al. The anti-atherosclerotic effect of tanshinol borneol ester using fecal metabolomics based on liquid chromatography-mass spectrometry. Analyst. 2016;141(3):1112-1120.

5. Wang J, Xu PF, Xie XN, et al. DBZ (Danshensu Bingpian Zhi), a novel natural compound derivative, attenuates atherosclerosis in apolipoprotein E-deficient mice. J Am Heart Assoc. 2017;6(10):e006297.

6. Rautio J, Kumpulainen H, Heimbach T, et al. Prodrugs: design and clinical applications. Nat Rev Drug Discov. 2008;7(3):255-270.

7. Liu D, Zheng XH, Tang YT, et al. Metabolism of tanshinol borneol ester in rat and human liver microsomes. Drug Metab Dispos. 2010;38(9): 1464-1470.

8. Liu D, Gao YW, Wang HJ, et al. Evaluation of the effects of cytochrome P450 nonsynonymous single-nucleotide polymorphisms on tanshinol borneol ester metabolism and inhibition potential. Drug Metab Dispos. 2010;38(12):2259-2265.

9. Pardridge WM. Drug transport across the blood-brain barrier. J Cereb Blood Flow Metab. 2012;32(11):1959-1972.

10. El-Helw ARM, Fahmy UA. Improvement of fluvastatin bioavailability by loading on nanostructured lipid carriers. Int J Nanomedicine. 2015;10:5797-5804.

11. Gupta B, Poudel BK, Tran TH, et al. Modulation of pharmacokinetic and cytotoxicity profile of imatinib base by employing optimized nanostructured lipid carriers. Pharm Res. 2015;32(9):2912-2927.

12. Arora R, Katiyar SS, Kushwah V, Jain S. Solid lipid nanoparticles and nanostructured lipid carrier-based nanotherapeutics in treatment of psoriasis: a comparative study. Expert Opin Drug Deliv. 2017;14(2): 165-177.

13. Balguri SP, Adelli GR, Majumdar S. Topical ophthalmic lipid nanoparticle formulations (SLN, NLC) of indomethacin for delivery to the posterior segment ocular tissues. Eur J Pharm Biopharm. 2016;109: 224-235. 
14. Jia LJ, Shen JY, Zhang DR, et al. In vitro and in vivo evaluation of oridonin-loaded long circulating nanostructured lipid carriers. Int J Biol Macromol. 2012;50(3):523-529.

15. Suk JS, Xu QG, Kim N, Hanes J, Ensign LM. PEGylation as a strategy for improving nanoparticle-based drug and gene delivery. Adv Drug Deliv Rev. 2016;99(Pt A):28-51.

16. Balguri SP, Adelli GR, Janga KY, Bhagav P, Majumdar S. Ocular disposition of ciprofloxacin from topical, PEGylated nanostructured lipid carriers: effect of molecular weight and density of poly(ethylene) glycol. Int J Pharm. 2017;529(1-2):32-43.

17. Schöttler $S$, Becker G, Winzen S, et al. Protein adsorption is required for stealth effect of poly(ethylene glycol)- and poly(phosphoester)-coated nanocarriers. Nat Nanotechnol. 2016;11(4):372-377.

18. Luan JJ, Zhang DR, Hao LL, et al. Preparation, characterization and pharmacokinetics of AmoitoneB-loaded long circulating nanostructured lipid carriers. Colloids Surf B: Biointerfaces. 2014;114(2): 255-260.

19. Joshi M, Patravale V. Nanostructured lipid carrier (NLC) based gel of celecoxib. Int J Pharm. 2008;346(1-2):124-132.

20. Zhang YJ, Yang JH, Han JJ, Dai GD. In vivo evaluation on transdermal delivery properties of matrine transfersomes by skin microdialysis method. Chin Tradit Herbal Drugs. 2013;44(23):3341-3345.

21. Zhao XX, Li JY, Cui YT, Sun Z, Zhang YJ. Assessment of tanshinone IIA self-microemulsifying preparation with drug release in vitro and intestinal absorption in situ. Chin J Tradit Chin Med Pharm. 2012; 27(8):2189-2191.

22. Stern ST, Zou P, Skoczen S, et al. Prediction of nanoparticle prodrug metabolism by pharmacokinetic modeling of biliary excretion. $J$ Control Release. 2013;172(2):558-567.

23. Liu SS, Ho PC. Intranasal administration of brain-targeted HP- $\beta-C D /$ chitosan nanoparticles for delivery of scutellarin, a compound with protective effect in cerebral ischaemia. J Pharm Pharmacol. 2017; 69(11):1495-1501.

24. Wiesmann M, Timmer NM, Zinnhardt B, et al. Effect of a multinutrient intervention after ischemic stroke in female C57B1/6 mice. $J$ Neurochem. Epub September 9, 2017.

25. Mukherjee B, Santra K, Pattnaik G, Ghosh S. Preparation, characterization and in-vitro evaluation of sustained release protein-loaded nanoparticles based on biodegradable polymers. Int J Nanomedicine. 2008; 3(4):487-496.

26. Zhao GR, Zhang HM, Ye TX, et al. Characterization of the radical scavenging and antioxidant activities of danshensu and salvianolic acid B. Food Chem Toxicol. 2008;46(1):73-81.

27. Han JY, Fan JY, Horie Y, et al. Ameliorating effects of compounds derived from Salvia miltiorrhiza root extract on microcirculatory disturbance and target organ injury by ischemia and reperfusion. Pharmacol Ther. 2008;117(2):280-295.

28. Wu L, Qiao H, Li Y, Li L. Protective roles of puerarin and danshensu on acute ischemic myocardial injury in rats. Phytomedicine. 2007; 14(10):652-658.
29. Guo C, Yin Y, Duan JL, et al. Neuroprotective effect and underlying mechanism of sodium danshensu (3-[3,4-dihydroxyphenyl] lactic acid from Radix and Rhizoma Salviae miltiorrhizae $=$ Danshen) against cerebral ischemia and reperfusion injury in rats. Phytomedicine. 2015; 22(2):283-289.

30. He XJ, Zhao LM, Liu YL. Effects of borneol injection on experimental cerebral ischemia in mice. West China J Pharm Sci. 2005;20: 323-325.

31. Zhao BS, Mi SQ. Effect of borneolum on expression of ICAM-1 in microvascular endothelial cells of rat brain. Tradit Chin Drug Res Clin Pharmacol. 2001;12(2):88-90.

32. Chen ZZ, Lu Y, Du SY, Shang KX, Cai CB. Influence of borneol and muscone on geniposide transport through MDCK and MDCK-MDR1 cells as blood-brain barrier in vitro model. Int J Pharm. 2013;456(1): 73-79.

33. Fan X, Chai LJ, Zhang H, Wang YF, Zhang BL, Gao XM. Borneol depresses P-glycoprotein function by a NF- $\mathrm{kB}$ signaling mediated mechanism in a blood brain barrier in vitro model. Int J Mol Sci. 2015;16(11): 27576-27588.

34. Khan AA, Mudassir J, Mohtar N, Darwis Y. Advanced drug delivery to the lymphatic system: lipid-based nanoformulations. Int J Nanomedicine. 2013;8:2733-2744.

35. Blanco E, Sangai A, Hsiao S, et al. Multistage delivery of chemotherapeutic nanoparticles for breast cancer treatment. Cancer Lett. 2013; 334(2):245-252.

36. Saneja A, Kumar R, Singh A, et al. Development and evaluation of long-circulating nanoparticles loaded with betulinic acid for improved anti-tumor efficacy. Int J Pharm. 2017;531(1):153-166.

37. Monopoli M, Åberg C, Salvati A, Dawson K. Biomolecular coronas provide the biological identity of nanosized materials. Nat Nanotechnol. 2012;7(12):779-786.

38. Wang XM, He HB, Leng W, Tang X. Evaluation of brain-targeting for the nasal delivery of estradiol by the microdialysis method. Int $J$ Pharm. 2006;317(1):40-46.

39. Nabavi SF, Dean OM, Turner A, Sureda A, Daglia M, Nabavi SM. Oxidative stress and post-stroke depression: possible therapeutic role of polyphenols? Curr Med Chem. 2015;22(3):343-351.

40. Perez-Pinzon MA, Dave KR, Raval AP. Role of reactive oxygen species and protein kinase $\mathrm{C}$ in ischemic tolerance in the brain. Antioxid Redox Signal. 2005;7(9-10):1150-1157.

41. Foley TD, Cantarella KM, Gillespie PF, Stredny ES. Protein vicinal thiol oxidations in the healthy brain: not so radical links between physiological oxidative stress and neural cell activities. Neurochem Res. 2014;39(11):2030-2039.

42. Miyamoto N, Maki T, Pham LDD, et al. Oxidative stress interferes with white matter renewal after prolonged cerebral hypoperfusion in mice. Stroke. 2013;44(12):3516-3521.
International Journal of Nanomedicine

\section{Publish your work in this journal}

The International Journal of Nanomedicine is an international, peerreviewed journal focusing on the application of nanotechnology in diagnostics, therapeutics, and drug delivery systems throughout the biomedical field. This journal is indexed on PubMed Central, MedLine, CAS, SciSearch ${ }^{\circledR}$, Current Contents ${ }^{\circledR} /$ Clinical Medicine,

\section{Dovepress}

Journal Citation Reports/Science Edition, EMBase, Scopus and the Elsevier Bibliographic databases. The manuscript management system is completely online and includes a very quick and fair peer-review system, which is all easy to use. Visit http://www.dovepress.com/ testimonials.php to read real quotes from published authors. 